Article

\title{
Some Symmetric Identities Involving Fubini Polynomials and Euler Numbers
}

\author{
Zhao Jianhong ${ }^{1}$ and Chen Zhuoyu ${ }^{2, *}$ \\ 1 Department of Teachers Education, Lijiang Teachers College, Lijiang 674199, China; zjh3004@163.com \\ 2 School of Mathematics, Northwest University, Xi'an 710127, China \\ * Correspondence: chenzymath@163.com
}

Received: 30 June 2018; Accepted: 24 July 2018; Published: 1 August 2018

\begin{abstract}
The aim of this paper is to use elementary methods and the recursive properties of a special sequence to study the computational problem of one kind symmetric sums involving Fubini polynomials and Euler numbers, and give an interesting computational formula for it. At the same time, we also give a recursive calculation method for the general case.
\end{abstract}

Keywords: Fubini polynomials; Euler numbers; symmetric identities; elementary method; computational formula

MSC: 11B83; 11B37

\section{Introduction}

For any integer $n \geq 0$, the Fubini polynomials $\left\{F_{n}(y)\right\}$ are defined by the coefficients of the generating function

$$
\frac{1}{1-y\left(e^{t}-1\right)}=\sum_{n=0}^{\infty} \frac{F_{n}(y)}{n !} \cdot t^{n}
$$

where $F_{0}(y)=1, F_{1}(y)=y$, and so on. $F_{n}(1)=F_{n}$ are called Fubini numbers. These polynomials and numbers are closely connected with the Stirling numbers. Some contents and propertities of Stirling numbers can be found in reference [1]. T. Kim et al. [2] proved the identity

$$
F_{n}(y)=\sum_{k=0}^{n} S_{2}(n, k) k ! y^{k},(n \geq 0),
$$

where $S_{2}(n, k)$ are the Stirling numbers of the second kind. It not only associated Fubini polynomials with Stirling numbers, but also stressed the importance of researching Fubini polynomials.

Please note that the identity (see [3,4])

$$
\frac{2 e^{t x}}{1+e^{t}}=\sum_{n=0}^{\infty} \frac{E_{n}(x)}{n !} \cdot t^{n}
$$

where $E_{n}(x)$ signifies the Euler polynomials.

It is distinct that if taking $y=-\frac{1}{2}$ in (1) and $x=0$ in (2), then from (1) and (2) we can get the identity

$$
E_{n}(0)=F_{n}\left(-\frac{1}{2}\right), n \in N^{*} 0
$$


where $E_{n}(0)=E_{n}$ is the Euler number (see [5] for related contents).

On the other hand, two variable Fubini polynomials are defined by means of the following (see $[2,6])$

$$
\frac{e^{x t}}{1-y\left(e^{t}-1\right)}=\sum_{n=0}^{\infty} \frac{F_{n}(x, y)}{n !} \cdot t^{n}
$$

and $F_{n}(y)=F_{n}(0, y)$ for all integers $n \geq 0$. About the properties of $F_{n}(x, y)$, several scholars have also researched it, especially T. Kim and others have done a large amount of vital works. For instance, they proved a series of identities linked to $F_{n}(x, y)$ (see [2,7]), one of which is

$$
F_{n}(x, y)=\sum_{l=0}^{n}\left(\begin{array}{c}
n \\
l
\end{array}\right) x^{l} \cdot F_{n-l}(y), n \in N^{*} 0
$$

These polynomials occupy indispensable positions in the theory and application of mathematics. In particular, they are widely used in combinatorial mathematics. Therefore, several scholars have researched their various properties, and acquired a series of vital results. Some involved contents can be found in references [5,7-17].

The goal of this paper is to use elementary methods and recursive properties of a special sequenc to research the computational problem of the sums

$$
\sum_{a_{1}+a_{2}+\cdots+a_{k}=n} \frac{F_{a_{1}}(y)}{\left(a_{1}\right) !} \cdot \frac{F_{a_{2}}(y)}{\left(a_{2}\right) !} \cdots \frac{F_{a_{k}}(y)}{\left(a_{k}\right) !}
$$

where the summation is over all $k$-tuples with non-negative integer coordinates $\left(a_{1}, a_{2}, \cdots, a_{k}\right)$ such that $a_{1}+a_{2}+\cdots+a_{k}=n$.

About this content, it seems there is no valid method to solve the computational problem of (4). However, this problem is significant, it can reveal the structure of Fubini polynomials itself and its internal relations, at least it can reflect the combination properties of Fubini polynomials.

In this paper, we will take elementary methods and the properties of $F_{n}(y)$ to obtain a fascinating computational formula for (4). Simultaneously, we can also acquire a recursive calculation method for the general case. That is, we are going to prove the following major result:

Theorem 1. For any positive integers $n$ and $k$, we have the identity

$$
\begin{aligned}
& \sum_{a_{1}+a_{2}+\cdots+a_{k}=n} \frac{F_{a_{1}}(y)}{\left(a_{1}\right) !} \cdot \frac{F_{a_{2}}(y)}{\left(a_{2}\right) !} \cdots \frac{F_{a_{k}}(y)}{\left(a_{k}\right) !} \\
= & \frac{1}{(k-1) !(y+1)^{k-1}} \cdot \frac{1}{n !} \sum_{i=0}^{k-1} C(k-1, i) F_{n+k-1-i}(y),
\end{aligned}
$$

where the sequence $\{C(k, i)\}$ is defined as follows: For any positive integer $k$ and integers $0 \leq i \leq k$, we define $C(k, 0)=1, C(k, k)=k !$ and

$$
C(k+1, i+1)=C(k, i+1)+(k+1) C(k, i), \text { for all } 0 \leq i<k,
$$

providing $C(k, i)=0$, if $i>k$.

The characteristic of this theorem is to represent a complex sum of Fubini polynomials as a linear combination of a single Fubini polynomial. Of course, our method can also be further generalized, provided a corresponding results for $F_{n}(x, y)$. It is just that its form is not so pretty, so we are not listing it here. If taking $k=3,4$ and 5 , then from our theorem we may instantly deduce the following several corollaries: 
Corollary 1. For any positive integer $n$, we have the identity

$$
\sum_{a+b+c=n} \frac{F_{a}(y)}{a !} \cdot \frac{F_{b}(y)}{b !} \cdot \frac{F_{c}(y)}{c !}=\frac{1}{2 \cdot n ! \cdot(y+1)^{2}}\left(F_{n+2}(y)+3 F_{n+1}(y)+2 F_{n}(y)\right) .
$$

Corollary 2. For any positive integer $n$, we have the identity

$$
\begin{aligned}
& \sum_{a+b+c+d=n} \frac{F_{a}(y)}{a !} \cdot \frac{F_{b}(y)}{b !} \cdot \frac{F_{c}(y)}{c !} \cdot \frac{F_{d}(y)}{d !} \\
= & \frac{1}{6 \cdot n ! \cdot(y+1)^{3}}\left(F_{n+3}(y)+6 F_{n+2}(y)+11 F_{n+1}(y)+6 F_{n}(y)\right) .
\end{aligned}
$$

Corollary 3. For any positive integer $n$, we have the identity

$$
\begin{aligned}
& \sum_{a+b+c+d+e=n} \frac{F_{a}(y)}{a !} \cdot \frac{F_{b}(y)}{b !} \cdot \frac{F_{c}(y)}{c !} \cdot \frac{F_{d}(y)}{d !} \cdot \frac{F_{e}(y)}{e !} \\
= & \frac{1}{24 \cdot n ! \cdot(y+1)^{4}}\left(F_{n+4}(y)+10 F_{n+3}(y)+35 F_{n+2}(y)+50 F_{n+1}(y)+24 F_{n}(y)\right) .
\end{aligned}
$$

If taking $y=-\frac{1}{2}$ in our theorem, then from (3) we can also infer the following:

Corollary 4. For any positive integers $n$ and $k \geq 2$, we have the identity

$$
\sum_{a_{1}+a_{2}+\cdots+a_{k}=n} \frac{E_{a_{1}}}{\left(a_{1}\right) !} \cdot \frac{E_{a_{2}}}{\left(a_{2}\right) !} \cdots \frac{E_{a_{k}}}{\left(a_{k}\right) !}=\frac{2^{k-1}}{(k-1) !} \cdot \frac{1}{n !} \sum_{i=0}^{k-1} C(k-1, i) E_{n+k-1-i} .
$$

If $n=p$ is an odd prime, then taking $y=1$ in Corollarys 1 and 2, we also have the following congruences.

Corollary 5. For any odd prime $p$, we have the congruence

$$
22 F_{p} \equiv F_{p+2}+3 F_{p+1}(\bmod p) .
$$

Corollary 6. For any odd prime $p$, we have the congruence

$$
186 F_{p} \equiv F_{p+3}+6 F_{p+2}+11 F_{p+1}(\bmod p) .
$$

\section{A Simple Lemma}

For purpose of proving our theorem, we need a uncomplicated lemma. As a matter of convenience, we first present a new sequence $\{C(k, i)\}$ as follows. For any positive integer $k$ and integers $0 \leq i \leq k$, we define $C(k, 0)=1, C(k, k)=k !$ and

$C(k+1, i+1)=C(k, i+1)+(k+1) C(k, i), 1 \leq i \leq k, C(k, i)=0$, if $i>k$.

For clarity, for $1 \leq k \leq 9$, we list values of $C(k, i)$ in the Table 1 . 
Table 1. Values of $C(k, i)$.

\begin{tabular}{|c|c|c|c|c|c|c|c|c|c|c|}
\hline$C(k, i)$ & $i=0$ & $i=1$ & $i=2$ & $i=3$ & $i=4$ & $i=5$ & $i=6$ & $i=7$ & $i=8$ & $i=9$ \\
\hline$k=1$ & 1 & 1 & & & & & & & & \\
\hline$k=2$ & 1 & 3 & 2 & & & & & & & \\
\hline$k=3$ & 1 & 6 & 11 & 6 & & & & & & \\
\hline$k=4$ & 1 & 10 & 35 & 50 & 24 & & & & & \\
\hline$k=5$ & 1 & 15 & 85 & 225 & 274 & 120 & & & & \\
\hline$k=6$ & 1 & 21 & 175 & 735 & 1624 & 1764 & 720 & & & \\
\hline$k=7$ & 1 & 28 & 322 & 1960 & 6769 & 13,132 & 13,068 & 5040 & & \\
\hline$k=8$ & 1 & 36 & 546 & 4536 & 22,449 & 67,284 & 118,124 & 109,584 & 40,320 & \\
\hline$k=9$ & 1 & 45 & 870 & 9450 & 63,273 & 269,325 & 723,680 & $1,172,700$ & $1,026,576$ & 362,880 \\
\hline
\end{tabular}

Obviously, the values of $C(k, i)$ can be easily calculated by using a computer program. Hence, for any positive integer $k$, the computational problem of (4) can be solved fully.

In this table of numerical values, we also find that for prime $p=3,5$ and 7 , we have the congruence

$$
C(p-1, i) \equiv 0(\bmod p) \text { for all } 1 \leq i \leq p-2 \text {. }
$$

For all prime $p>7$ is true? This is an enjoyable open problem.

If this congruence is true, then we can also deduce that for any positive integer $n$ and odd prime $p$, one has the congruence

$$
F_{n+p-1}(y)+F_{n}(y) \equiv 0(\bmod p) .
$$

Now let function $f(t)=\frac{1}{1-y\left(e^{t}-1\right)}$. Then we have the following

Lemma 1. For any positive integer $k$, we have the identity

$$
\sum_{i=0}^{k} C(k, i) f^{(k-i)}(t)=k !(y+1)^{k} f^{k+1}(t),
$$

where $f^{(0)}(t)=f(t), f^{(r)}(t)$ denotes the r-order derivative of $f(t)$ for variable $t$.

Proof. Now we prove this lemma by induction. From the definition of the derivative we acquire

$$
f^{\prime}(t)=\frac{y e^{t}}{\left(1-y\left(e^{t}-1\right)\right)^{2}}=-f(t)+(y+1) f^{2}(t)
$$

or

$$
f^{\prime}(t)+f(t)=(y+1) f^{2}(t)
$$

Please note that $C(1,0)=1$ and $C(1,1)=1$, so the lemma is true for $k=1$.

Suppose that the lemma is true for all integer $k \geq 1$. That is,

$$
\sum_{i=0}^{k} C(k, i) f^{(k-i)}(t)=k !(y+1)^{k} f^{k+1}(t)
$$

Then take the derivative for $t$ in (7) and applying (5) and (7) we obtain

$$
\begin{aligned}
& \sum_{i=0}^{k} C(k, i) f^{(k+1-i)}(t)=(k+1) !(y+1)^{k} f^{k}(t) \cdot f^{\prime}(t) \\
= & (k+1) !(y+1)^{k} f^{k}(t) \cdot\left(-f(t)+(y+1) f^{2}(t)\right) \\
= & (k+1) !(y+1)^{k+1} f^{k+2}(t)-(k+1) !(y+1)^{k} f^{k+1}(t) \\
= & (k+1) !(y+1)^{k+1} f^{k+2}(t)-(k+1)\left(\sum_{i=0}^{k} C(k, i) f^{(k-i)}(t)\right) .
\end{aligned}
$$


It is evident that (8) implies

$$
\begin{aligned}
& (k+1) !(y+1)^{k+1} f^{k+2}(t) \\
= & C(k, 0) f^{(k+1)}(t)+\sum_{i=0}^{k-1}(C(k, i+1)+(k+1) C(k, i)) f^{(k-i)}(t)+(k+1) ! f(t) \\
= & C(k, 0) f^{(k+1)}(t)+\sum_{i=0}^{k-1} C(k+1, i+1) f^{(k-i)}(t)+(k+1) ! f(t) \\
= & \sum_{i=0}^{k+1} C(k+1, i) f^{(k+1-i)}(t),
\end{aligned}
$$

where we have used the identities $C(k, 0)=1$ and $C(k, k)=k$ !. Now the lemma follows from (9) and mathematical induction.

\section{Proof of the Theorem}

In this section, the proof of our theorem will be completed. Firstly, for any positive integer $k$, from the definition of $f(t)$ and the properties of the power series we obtain

$$
f^{(k)}(t)=\sum_{n=0}^{\infty} \frac{F_{n+k}(y)}{n !} \cdot t^{n}
$$

and

$$
\begin{aligned}
& f^{k}(t)=\left(\sum_{a_{1}=0}^{\infty} \frac{F_{a_{1}}(y)}{a_{1} !} \cdot t^{a_{2}}\right)\left(\sum_{a_{2}=0}^{\infty} \frac{F_{a_{2}}(y)}{a_{2} !} \cdot t^{a_{1}}\right) \cdots\left(\sum_{a_{k}=0}^{\infty} \frac{F_{a_{k}}(y)}{a_{k} !} \cdot t^{a_{k}}\right) \\
= & \left(\sum_{a_{1}=0}^{\infty} \sum_{a_{2}=0}^{\infty} \cdots \sum_{a_{k}=0}^{\infty} \frac{F_{a_{1}}(y)}{\left(a_{1}\right) !} \cdot \frac{F_{a_{2}}(y)}{\left(a_{2}\right) !} \cdots \frac{F_{a_{k}}(y)}{\left(a_{k}\right) !} \cdot t^{a_{1}+a_{2} \cdots+a_{k}}\right) \\
= & \sum_{n=0}^{\infty}\left(\sum_{a_{1}+a_{2}+\cdots+a_{k}=n} \frac{F_{a_{1}}(y)}{\left(a_{1}\right) !} \cdot \frac{F_{a_{2}}(y)}{\left(a_{2}\right) !} \cdots \frac{F_{a_{k}}(y)}{\left(a_{k}\right) !}\right) \cdot t^{n} .
\end{aligned}
$$

From (10), (11) and Lemma we acquire

$$
\begin{aligned}
& \frac{1}{(k-1) !(y+1)^{k-1}} \cdot \sum_{i=0}^{k-1} C(k-1, i) \sum_{n=0}^{\infty} \frac{F_{n+k-1-i}(y)}{n !} \cdot t^{n} \\
& =\sum_{n=0}^{\infty}\left(\sum_{a_{1}+a_{2}+\cdots+a_{k}=n} \frac{F_{a_{1}}(y)}{\left(a_{1}\right) !} \cdot \frac{F_{a_{2}}(y)}{\left(a_{2}\right) !} \cdots \frac{F_{a_{k}}(y)}{\left(a_{k}\right) !}\right) \cdot t^{n} \text {. }
\end{aligned}
$$

Comparing the coefficients of $t^{n}$ in (12) we have the identity

$$
\begin{aligned}
& \sum_{a_{1}+a_{2}+\cdots+a_{k}=n} \frac{F_{a_{1}}(y)}{\left(a_{1}\right) !} \cdot \frac{F_{a_{2}}(y)}{\left(a_{2}\right) !} \cdots \frac{F_{a_{k}}(y)}{\left(a_{k}\right) !} \\
= & \frac{1}{(k-1) !(y+1)^{k-1}} \cdot \frac{1}{n !} \sum_{i=0}^{k-1} C(k-1, i) F_{n+k-1-i}(y) .
\end{aligned}
$$

This completes the proof of our Theorem.

Author Contributions: Writing-original draft: J.Z.; Writing-review and editing: Z.C.

Funding: This research was funded by the N. S. F. (11771351) of China.

Acknowledgments: The author would like to thank the referees for their very helpful and detailed comments, which have significantly improved the presentation of this paper.

Conflicts of Interest: The authors declare no conflict of interest. 


\section{References}

1. Feng, R.-Q.; Song, C.-W. Combinatorial Mathematics; Beijing University Press: Beijing, China, 2015.

2. Kim, T.; Kim, D.S.; Jang, G.-W. A note on degenerate Fubini polynomials. Proc. Jangjeon Math. Soc. 2017, 20, 521-531.

3. Kim, T. Symmetry of power sum polynomials and multivariate fermionic $p$-adic invariant integral on $Z_{p}$. Russ. J. Math. Phys. 2009, 16, 93-96. [CrossRef]

4. Kim, D.S.; Park, K.H. Identities of symmetry for Bernoulli polynomials arising from quotients of Volkenborn integrals invariant under $S_{3}$. Appl. Math. Comput. 2013, 219, 5096-5104. [CrossRef]

5. Zhang, W. Some identities involving the Euler and the central factorial numbers. Fibonacci Q. 1998, 36, 154-157.

6. Kilar, N.; Simesk, Y. A new family of Fubini type numbrs and polynomials associated with Apostol-Bernoulli nujmbers and polynomials. J. Korean Math. Soc. 2017, 54, 1605-1621.

7. Kim, T.; Kim, D. S.; Jang, G.-W.; Kwon, J. Symmetric identities for Fubini polynomials. Symmetry 2018, 10, 219. [CrossRef]

8. Kim, T.; Kim, D.S. An identity of symmetry for the degernerate Frobenius-Euler polynomials. Math. Slovaca 2018, 68, 239-243. [CrossRef]

9. He, Y. Symmetric identities for Calitz's q-Bernoulli numbers and polynomials. Adv. Differ. Equ. 2013, $2013,246$. [CrossRef]

10. Rim, S.-H.; Jeong, J.-H.; Lee, S.-J.; Moon, E.-J.; Jin, J.-H. On the symmetric properties for the generalized twisted Genocchi polynomials. ARS Comb. 2012, 105, 267-272.

11. Yi, Y.; Zhang, W. Some identities involving the Fibonacci polynomials. Fibonacci Q. 2002, 40, 314-318.

12. Ma, R.; Zhang, W. Several identities involving the Fibonacci numbers and Lucas numbers. Fibonacci Q. 2007, $45,164-170$.

13. Wang, T.; Zhang, W. Some identities involving Fibonacci, Lucas polynomials and their applications. Bull. Math. Soc. Sci. Math. Roum. 2012, 55, 95-103.

14. Chen, L.; Zhang, W. Chebyshev polynomials and their some interesting applications. Adv. Differ. Equ. 2017, $2017,303$.

15. Li, X.X. Some identities involving Chebyshev polynomials. Math. Probl. Eng. 2015, 2015, 950695. [CrossRef]

16. Ma, Y.; Lv, X.-X. Several identities involving the reciprocal sums of Chebyshev polynomials. Math. Probl. Eng. 2017, 2017, 4194579. [CrossRef]

17. Clemente, C. Identities and generating functions on Chebyshev polynomials. Georgian Math. J. 2012, 19, $427-440$.

(C) 2018 by the authors. Licensee MDPI, Basel, Switzerland. This article is an open access article distributed under the terms and conditions of the Creative Commons Attribution (CC BY) license (http:// creativecommons.org/licenses/by/4.0/). 